\title{
Efecto del sorbitol sobre la relajación estructural en películas de gelatina en estado vítreo
}

\author{
Effect of sorbitol on structural relaxation of \\ gelatin films in the glassy state
}

\section{Efeito do sorbitol em relaxação estrutural de filmes de gelatina em estado vítreo}

\author{
Paulo Díaz-Calderón'; Franck Quero; Bill MacNaughtan²; Mina Rousennova ${ }^{3}$; Javier Enrione ${ }^{1 *}$ \\ ${ }^{1}$ Biopolymer Research and Engineering Lab (BiopREL), Escuela de Nutrición y Dietética, Universidad de los \\ Andes. M. Alvaro del Portillo 12.455, Las Condes, Santiago, Chile. \\ 2Division of Food Sciences, The University of Nottingham. Sutton Bonington Campus, Loughbourough LE12 5RD, \\ Reino Unido. \\ ${ }^{3}$ School of Physics. HH Wills Physics Laboratory, University of Bristol, Tyndall Avenue BS8 1TL, Bristol, Reino \\ Unido. \\ *jenrione@uandes.cl
}

Fecha Recepción: 23 de diciembre de 2014 Fecha Aceptación: 26 de junio de 2015

\begin{abstract}
Resumen
El objetivo de este trabajo fue evaluar el efecto del sorbitol sobre la cinética de relajación estructural de películas de gelatina almacenadas bajo la temperatura de transición vítrea (Tg). Películas de gelatina de bovino y sorbitol fueron preparadas mediante casting en frío. El sorbitol fue agregado en fracciones en peso (Qs) de 0,0, 0,06 y 0,10. Las películas fueron acondicionadas en un ambiente de humedad relativa constante (44\%) utilizando una solución saturada de carbonato de potasio, obteniéndose fracciones de contenido de humedad en peso $(\mathrm{Qw})$ de $0,18,0,16$ y 0,18 respectivamente. La entalpía de relajación $(\Delta \mathrm{H})$ fue determinada mediante Calorimetría Diferencial de Barrido (DSC). Las muestras utilizadas en este estudio presentaron valores de $\mathrm{Tg}$ de $48^{\circ} \mathrm{C}(\mathrm{Qs}=0,0), 35^{\circ} \mathrm{C}(\mathrm{Qs}=0,06)$ y $30^{\circ} \mathrm{C}(\mathrm{Qs}=0,10)$. Luego de eliminar el historial térmico $\left(30^{\circ} \mathrm{C}\right.$ sobre $\left.\mathrm{Tg}, 15 \mathrm{~min}\right)$, las muestras fueron almacenadas isotérmicamente a $10^{\circ} \mathrm{C}$ bajo $\mathrm{Tg}_{\text {onset }}$ entre 2 y 80 horas. La adición de sorbitol produjo una reducción significativa $(\mathrm{p}<0,05)$ en la cinética de relajación estructural. La linealización del valor de entalpía de relajación $(\Delta \mathrm{H})$ en función del logaritmo del tiempo de almacenamiento mostró una reducción de la pendiente en las muestras plastificadas con sorbitol. La reducción en la cinética de relajación estaría relacionada con el efecto de empaquetamiento molecular asociado a la presencia de polioles en matrices en estado vítreo recientemente reportada mediante espectroscopía de positrones (PALS).
\end{abstract}

Palabras clave: Gelatina, plastificantes, estado vitreo, relajación entálpica.

\begin{abstract}
The objective of this work was to study the effect of sorbitol on the kinetic of enthalpy relaxation of gelatin films stored at temperatures below the glass transition temperature ( $\mathrm{Tg}$ ). Bovine gelatin and sorbitol films were prepared by cold casting method. Sorbitol was added at weight fraction (Qs) of 0.0 , 0.06 and 0.10 . Films were equilibrated in environment of $44 \%$ relative humidity using a saturated salt solution of potassium carbonate, giving a moisture weight fraction in samples $(\mathrm{Qw})$ of $0.18,0.16$ and 0.18 respectively. Enthalpy relaxation $(\Delta \mathrm{H})$ was measured by differential scanning calorimetry (DSC). Tg


values of the samples tested were observed at $48^{\circ} \mathrm{C}(\mathrm{Qs}=0.0), 35^{\circ} \mathrm{C}(\mathrm{Qs}=0.06)$ and $30^{\circ} \mathrm{C}(\mathrm{Qs}=0.10)$. After the thermal history deletion $\left(30^{\circ} \mathrm{C}\right.$ above $\mathrm{Tg}, 15$ minutes), samples were annealed from 2 to 80 hours at $10^{\circ} \mathrm{C}$ below $\mathrm{Tg}_{\text {onset }}$. The addition of sorbitol showed a significant reduction $(\mathrm{p}<0.05)$ in structural relaxation kinetic. The linearization of relaxation enthalpy $(\Delta \mathrm{H})$ versus log of annealing time showed a reduction of the slope in samples plasticized with sorbitol. The reduction in structural relaxation kinetic could be related to the effect of molecular packing by the presence of polyol in polymer matrix as has been recently reported by Positron Annihilation Spectroscopy (PALS).

Keywords: gelatin, plasticizers, glassy state, physical aging.

\section{Resumo}

O objetivo deste estudo foi avaliar o efeito do sorbitol sobre a cinética da relaxação estrutural de películas de gelatina armazenadas a temperatura inferior a temperatura de transição vítrea $(\mathrm{Tg})$. Filmes de gelatina bovina e sorbitol foram preparados por moldagem a frio. O sorbitol foi adicionado em fracções de peso (QS), de 0,0, 0,06 e 0,10. Os filmes foram acondicionados em atmosfera de humidade relativa constante (44\%), utilizando uma solução saturada de carbonato de potássio, obtendo-se de frações de teor de humidade de peso $(\mathrm{Qw})$ de 0,18, 0,16 e 0,18, respectivamente. A entalpia de relaxação $(\Delta \mathrm{H})$ foi determinada por Calorimetria Diferencial de Varrimento (DSC). As amostras usadas neste estudo apresentaram valores de $\mathrm{Tg}$ de $48^{\circ} \mathrm{C}(\mathrm{Qs}=0,0), 35^{\circ} \mathrm{C}(\mathrm{Qs}=0,06)$ e $30^{\circ} \mathrm{C}(\mathrm{Qs}=0,10)$. Após eliminar historial térmico $\left(30^{\circ} \mathrm{C}\right.$ a Tg, 15 minutos), as amostras foram armazenadas isotermicamente a $10^{\circ} \mathrm{C}$ sob $\operatorname{Tg}_{\text {onset }}$ entre 2 e 80 horas. A adição de sorbitol produziu uma redução significativa $(p<0,05)$ na cinética da relaxação estrutural. A linearização do valor de entalpia de relaxação $(\Delta \mathrm{H})$ versus o logaritmo do tempo de armazenamento apresentou uma redução do declive nas amostras plastificados com sorbitol. A redução da cinética de relaxamento poderia estar relacionada com o efeito de empaquetamiento molecular associado à presença de polióis em matrizes em estado vítreo recentemente relatado pela espectroscopia de positroês (PALS).

Palabras-chave: gelatina, plastificantes, estado vitreo, relaxação estrutural.

\section{Introducción}

La estabilidad estructural de un material polimérico es dependiente del estado físico de la matriz (ordenamiento molecular), del contenido de sólidos (o contenido de humedad) y de la temperatura. Así un material sólido molecularmente ordenado, como es el caso de un cristal, tendrá niveles de estabilidad distintos en comparación con aquellos materiales amorfos o molecularmente desordenados, caracterizados por no poseer un ordenamiento regular a escala molecular con lo cual la posición exacta de una cierta molécula en un tiempo determinado no puede ser definido $[1,2]$. Estructuras amorfas se obtienen en una serie de procesos en la industria de alimentos, tales como horneado, concentración, liofilización, secado spray y extrusión, en los cuales la etapa de remoción de humedad o reducción de temperatura (congelación) ocurre en tiempos cortos $[3,4]$. Durante el almacenamiento un material polimérico busca alcanzar una condición de equilibrio termodinámico en función del ambiente que lo rodea, y lo hace mediante ajustes estructurales que están determinados por el estado físico de la matriz, la humedad y por la temperatura. En el caso de materiales poliméricos amorfos almacenados bajo la temperatura de transición vítrea $(\mathrm{Tg})$, la movilidad molecular se encuentra fuertemente restringida debido a la alta viscosidad del sistema (aproximadamente $\left.10^{12} \mathrm{~Pa} . \mathrm{s}^{1}\right)[5,6,7]$. Bajo estas condiciones el material tiende a alcanzar el equilibrio termodinámico mediante cambios micro-estructurales que están asociados con una liberación de energía $(\Delta \mathrm{H})$ y disminución del volumen específico (densificación) en un fenómeno conocido como relajación estructural o physical aging $[8,9]$. En un gráfico temperatura versus entalpía (o volumen específico, Figura 1) la relajación estructural se representa por la flecha que une los puntos A y B, a una cierta temperatura de almacenamiento Ta. Cabe señalar que este es un fenómeno espontáneo que ha sido ampliamente estudiado en polímeros sintéticos [1016], pero que requiere de mayor investigación en el área de biomateriales debido a las implicancias tecnológicas que éste posee. Durante un proceso de relajación se observan cambios significativos en algunas propiedades físicas del material, 
como por ejemplo aumento del módulo elástico [17-19], aumento de fragilidad [20] y disminución en los valores de permeabilidad y difusividad [21-24]. Este comportamiento estaría gobernado por la modificación estructural asociada con una organización molecular más compacta y un incremento en el grado de interacción molecular producto del fenómeno de relajación [9].

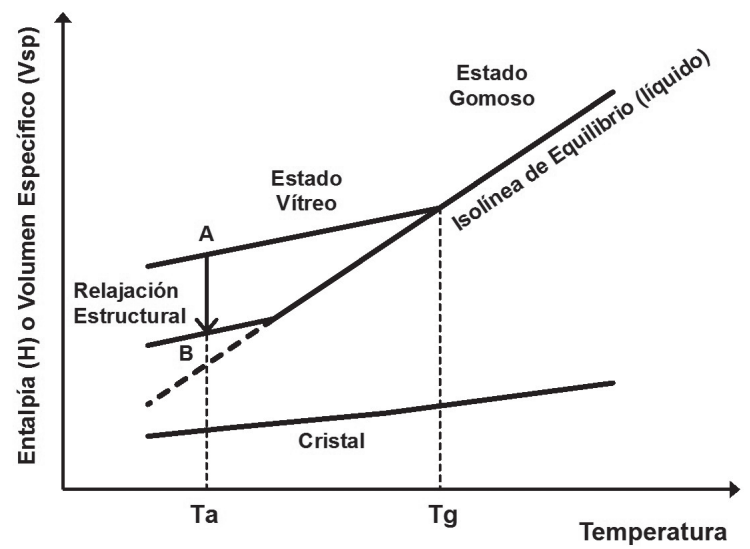

Figura 1. Cambios de entalpía y volumen específico durante un proceso de almacenamiento isotérmico en estado vítreo ( $\mathrm{T}<\mathrm{Tg})$, modificado de referencia [8].

La literatura científica ha mostrado que la presencia de compuestos de bajo peso molecular (plastificantes) en una matriz polimérica en estado vítreo produce importantes cambios estructurales, los cuales a escala macroscópica están asociados con una disminución del volumen específico de la matriz. Este comportamiento ha sido reportado en matrices poliméricas amorfas a base de maltodextrinas de distinto grado de dextrosa equivalente (DE) [25], maltopolímero-maltosa [26] y maltodextrinas de alto DE y glicerol [27] utilizando la técnica de picnometría de gas. Se ha reportado un comportamiento similar en películas semicristalinas de gelatina de bovino plastificadas con diferentes niveles de glicerol haciendo uso de la misma técnica [28]. Este comportamiento no deja de ser sorprendente ya que la literatura clásica en ciencias de materiales señala que la presencia de plastificantes produce un aumento de la movilidad molecular del sistema, fenómeno que estaría gobernado por un aumento del espacio intermolecular (o volumen libre) y una disminución de la viscosidad a nivel local [29-31]. Este fenómeno debiera estar asociado con un aumento del volumen específico del sistema, lo cual explicaría el hecho de que los plastificantes se utilicen en la industria para aumentar la flexibilidad y la extensibilidad de materiales poliméricos, facilitando su manejo a nivel de proceso [1]. Sin embargo, estudios a nivel nano-estructural utilizando la técnica de espectroscopía de positrones (Positron Annihilation Lifetime Spectroscopy, PALS) han reportado que a escala sub-nanométrica un plastificante produce una disminución en el volumen de los espacios libres $(\mathrm{Vh})$ existentes entre cadenas poliméricas, generando un aumento en el grado de empaquetamiento molecular [32,33,26]. En sistemas amorfos estos espacios libres se generan debido a un ordenamiento molecular irregular que es característico de materiales estructuralmente desordenados (amorfos). En este contexto se ha propuesto que la disminución de Vh se debería a la capacidad que tienen los compuestos de bajo peso molecular de ocupar estos volúmenes vacíos de dimensiones subnanométricas [27]. Este comportamiento ha sido descrito en matrices poliméricas amorfas en estado vítreo a base de maltodextrinas de distinto DE [32,25], en mezclas maltopolímeromaltosa $[33,26]$ y maltodextrinas (DE12)-glicerol [27]. Estudios recientes han reportado el efecto de empaquetamiento molecular por adición de glicerol y sorbitol en películas amorfas a base de gelatina de bovino en estado vítreo [34-36]. Estos cambios nano-estructurales ayudarían a explicar la disminución en la sorción de humedad que se observa en biomateriales en estado vítreo en presencia de plastificantes [37-40,28], donde se ha propuesto que en la medida que una matriz polimérica es más densa y compacta más difícil es para una molécula de agua interactuar con los sitios de sorción de la matriz, los cuales en estas condiciones además estarían siendo ocupados por el plastificante $[39,27]$. Por otra parte, se ha reportado una fuerte dependencia del valor de $\mathrm{Vh}$ respecto de la temperatura con un claro punto de inflexión a temperatura igual a $\mathrm{Tg}$, reportándose además una muy buena correlación entre los valores de $\mathrm{Tg}$ obtenidos mediante PALS con aquellos calculados mediante calorimetría (DSC) $[27,34,36]$.

Por lo tanto el efecto de empaquetamiento molecular que se produce por la presencia de compuestos de bajo peso molecular en una matriz polimérica en estado vítreo sugiere que la presencia de plastificantes podría tener un efecto significativo sobre la cinética de relajación estructural que ocurre bajo $\mathrm{Tg}$. El objetivo de este trabajo fue estudiar el efecto del sorbitol (plastificante de uso común en la industria de alimentos y farmacéutica) sobre la cinética de relajación estructural (physical aging) de gelatina de bovino en estado vítreo. 


\section{Metodología}

Películas de gelatina de bovino (Rousselot, Bloom 220, Brasil) y sorbitol (Blumos, peso molecular $182,17 \mathrm{~g} / \mathrm{mol}$, Chile) fueron preparadas mediante la técnica de casting en frío $\left(5^{\circ} \mathrm{C}\right)[19,28]$. A una suspensión de gelatina $(70 \mathrm{~g} / \mathrm{L})$ se agregó sorbitol en fracciones en peso (Qs) de 0,0, 0,06 y 0,10. Las muestras fueron depositadas sobre moldes de teflón y mantenidas a $5^{\circ} \mathrm{C}$ hasta obtener una película de espesor uniforme (aproximadamente $0,3 \mathrm{~mm}$ ). Las películas fueron mantenidas en un ambiente con aproximadamente $0 \%$ humedad relativa utilizando $\mathrm{P}_{2} \mathrm{O}_{5}$ durante una semana. Luego fueron acondicionadas en ambientes de humedad relativa constante $(44 \%$, solución saturada de $\mathrm{K}_{2} \mathrm{CO}_{3}$ ) hasta alcanzar el equilibrio gravimétrico, esto es, hasta que la diferencia en peso entre dos medidas consecutivas fuera menor al $0,5 \%$.

El valor de $\mathrm{Tg}$ de cada muestra se determinó mediante calorimetría diferencial de barrido (Pyris Diamond DSC, Perkin Elmer, USA). El equipo fue calibrado utilizando Indio como estándar $\left(\mathrm{Tm}=156,6^{\circ} \mathrm{C}\right.$ y $\left.\Delta \mathrm{H}=28,4 \mathrm{~J} / \mathrm{g}\right)$. Aproximadamente $20 \mathrm{mg}$ de muestra fueron selladas herméticamente en cápsulas de acero inoxidable de $60 \mu \mathrm{L}$, mientras que una cápsula vacía fue utilizada como referencia. La metodología utilizada fue la siguiente: calentamiento desde $-20^{\circ} \mathrm{C}$ hasta $120^{\circ} \mathrm{C}$ con una rampa térmica de $10^{\circ} \mathrm{C} / \mathrm{min}$, posteriormente se mantuvo a $120^{\circ} \mathrm{C}$ por 2 minutos, enfriamiento desde 120 a $-20^{\circ} \mathrm{C}$ a $40^{\circ} \mathrm{C} / \mathrm{min}$, se mantuvo a $-20^{\circ} \mathrm{C}$ por 2 minutos y calentamiento desde $-20^{\circ} \mathrm{C}$ hasta $120^{\circ} \mathrm{C}$ a $10^{\circ} \mathrm{C} / \mathrm{min}$. La $\mathrm{Tg}$ se consideró como el valor de temperatura en el onset de la transición asociada al cambio en la capacidad calorífica (Cp) observada en el segundo barrido de temperatura. Todas las muestras fueron analizadas en triplicado. La cinética de relajación entálpica (physical aging) fue determinada mediante DSC luego de eliminar el historial térmico de las muestras. Para ello las muestras fueron mantenidas a una temperatura constante $\mathrm{Te}\left(\mathrm{Te}=\mathrm{Tg}_{\text {endset }}+30^{\circ} \mathrm{C}\right)$ durante 15 minutos [41,42]. Luego las muestras fueron almacenadas durante 2, 4, 8, 16, 40 y 80 horas a una temperatura constante $\mathrm{Ta}\left(\mathrm{Ta}=\mathrm{Tg}_{\text {onset }}-10^{\circ} \mathrm{C}\right)$. Dos barridos térmicos fueron realizados entre $-10^{\circ} \mathrm{C}$ y $65^{\circ} \mathrm{C}$ a $10^{\circ} \mathrm{C} / \mathrm{min}$. La entalpía de relajación $\left(\Delta \mathrm{H}, \mathrm{J} / \mathrm{g}_{\text {muestra seca }}\right)$ fue calculada de la sustracción del primer barrido de temperatura respecto del segundo. Todas las muestras fueron analizadas en triplicado. Para permitir la comparación entre los termogramas obtenidos, las curvas fueron normalizadas siguiendo la metodología propuesta por Badii [49], en la cual se considera una temperatura de la zona gomosa del termograma ( $\mathrm{T}>\mathrm{Tg}$ ) y se normaliza respecto de la diferencia constante que existe entre un valor de flujo de calor en la zona gomosa y un valor extrapolado en la zona vítrea del termograma $(\mathrm{T}<\mathrm{Tg})$.

La medición del espacio libre intermolecular $(\mathrm{Vh})$ se desarrolló mediante espectroscopia de positrones PALS (self-assembled equipment,, School of Physics, University of Bristol, UK). Se utilizó un equipo PALS operado mediante un sistema fastfast coincidence con una función de resolución gaussiana (FWHN) de 0,175ns. El ${ }^{22} \mathrm{Na}$ fue utilizado como fuente de positrones y se preparó depositando dos gotas de ${ }^{22} \mathrm{NaCl}$ entre dos láminas de Kapton foil de 7,5 $\mu \mathrm{m}$. Cada corrida experimental fue desarrollada durante dos horas para generar al menos 2,5 millones de cuentas experimentales. El espectro de tiempo de vida media de los positrones fue analizado descomponiendo el espectro en tres componentes usando el software LT 9.1. El tamaño promedio de $\mathrm{Vh}$ fue calculado desde el tiempo de vida media del orto-positronium (o-Ps) usando la ecuación semi-empírica de Tau-Eldrup [43,44].

El análisis de significancia estadística fue realizado mediante ANOVA (Excel Office 2010, Microsoft Corp.).

\section{Resultados y Discusión}

La caracterización inicial de las películas de gelatina mediante DSC mostró que el sorbitol produce una disminución significativa del valor de $\mathrm{Tg}(p<0,05$, ANOVA) en todos los niveles de plastificante estudiados (Tabla 1). El sorbitol tiene un reconocido efecto reductor de $\mathrm{Tg}$ el cual ha sido ampliamente descrito en la literatura [45$48,40]$. El contenido de humedad de las películas disminuyó significativamente ( $p<0,05$, ANOVA) por presencia del sorbitol, sin embargo no se observaron diferencias significativas ( $p>0,05$, ANOVA) en el contenido de humedad entre los distintos niveles de sorbitol estudiados. Este comportamiento podría ser explicado por diferencias en el grado de interacción entre el sorbitol y la matriz de gelatina.

La Figura 2 presenta los termogramas obtenidos mediante DSC de las películas de gelatina control $(\mathrm{Qg}=0,00$, Figura $2 \mathrm{a})$ y con sorbitol $(\mathrm{Qg}=0,10$, Figura $2 \mathrm{~b})$, almacenados isotérmicamente $10^{\circ} \mathrm{C}$ bajo $\mathrm{Tg}_{\text {onset }}$ entre 0 y 80 horas. En ambas condiciones se observa claramente la presencia del fenómeno de 
relajación estructural debido a la presencia de un peak endotérmico situado en la zona del termograma donde se localiza la transición vítrea, el cual aumenta gradualmente a medida que aumenta el tiempo de almacenamiento. Esta endoterma es característica del proceso de relajación y se produce debido a que durante la etapa de calentamiento en un experimento mediante DSC, el material recupera la energía liberada durante el almacenamiento [21,8]. Esta energía se evalúa como el área bajo la endoterma y corresponde a una entalpía de relajación $(\Delta \mathrm{H}, \mathrm{J} /$ $\left.g_{\text {muestra seca }}\right)$.

Tabla 1. Contenido de humedad y $\mathrm{Tg}$ de películas gelatina-sorbitol*.

\begin{tabular}{ccc}
\hline $\begin{array}{c}\text { Contenido de Sorbitol } \\
(\mathbf{Q s})\end{array}$ & $\begin{array}{c}\text { Contenido de Humedad } \\
(\mathbf{Q w})\end{array}$ & $\begin{array}{c}\mathbf{T g}_{\text {onset }} \\
\left({ }^{\circ} \mathbf{C}\right)\end{array}$ \\
\hline 0,00 & $0,18(0,00014)$ & $49,1(0,3)$ \\
0,06 & $0,16(0,055)$ & $35,6(0,4)$ \\
0,10 & $0,16(0,013)$ & $29,4(1,4)$ \\
\hline
\end{tabular}

*Los datos presentados corresponden a la media $(n=3)$ y los valores en paréntesis a la desviación estándar. Letras iguales dentro de la misma columna indican diferencias no significativas $(p>0,05)$
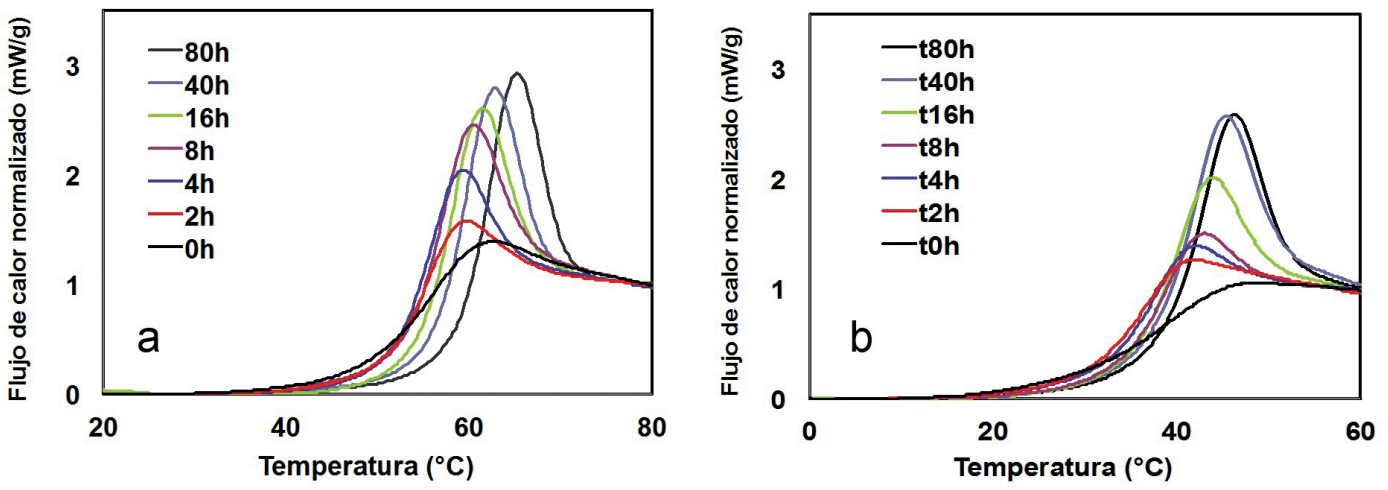

Figura 2. Relajación entálpica en películas de gelatina (a) y con sorbitol 0,10 (b) a distintos tiempos de almacenamiento (horas).

En la Figura 2 ( $a$ y b) es posible observar además dos comportamientos que son característicos del fenómeno de relajación estructural: i) a tiempos largos tanto la altura del pico como la entalpía de relajación tienden a alcanzar un valor constante (Figura 3a) y, ii) la temperatura a la cual se ubica el máximo del pico de la endoterma se desplaza hacia valores mayores de temperatura a medida que aumenta el tiempo de almacenamiento (Figura 3b). Ambos fenómenos se relacionan con la condición de no-linealidad de la relajación estructural y responden por un lado al fenómeno de disminución del volumen específico que ocurre a medida que el material relaja, y a la disminución de la movilidad molecular producto de la relajación que hace que la recuperación de energía se produzca a una temperatura más alta $[13,14]$.

Las Figuras $2 b$ y $3 a$ muestran el efecto del sorbitol sobre la cinética de relajación estructural de las películas de gelatina evaluada mediante DSC. Se observa que la presencia de sorbitol (independiente de la concentración) produce una disminución de los valores de entalpía de relajación para todos los tiempos de almacenamiento estudiados, lo cual sugiere que la presencia de sorbitol modifica la cinética de relajación estructural de gelatina cuando es almacenada bajo Tg. Este comportamiento se observa más claramente a tiempos largos de almacenamiento ( $\mathrm{t}>2 \mathrm{~h}$ ) y cuando los valores de entalpía de relajación son graficados en coordenadas semilogarítmicas. Tal como se observa en la Figura 4, la presencia de sorbitol produce una disminución significativa $(p<0,05$, ANOVA) en el valor de la pendiente de la curva $\Delta H$ vs $\log (\mathrm{t})$ en las películas de gelatina plastificadas con sorbitol (Qs 0,06 y 0,10 ) respecto de la muestra control (Qs 0,00). Sin embargo no se 
observan diferencias significativas $(p>0,05)$ entre los valores de entalpía de relajación obtenidos con distintos niveles de sorbitol.

Una posible explicación para este fenómeno estaría en los cambios nano-estructurales descritos en películas de gelatina-plastificante en estado vítreo mediante la técnica de Espectroscopía de Positrones (PALS). Estudios recientes han reportado que en el estado vítreo moléculas de bajo peso molecular, tales como glicerol y sorbitol, actúan aumentando el grado de empaquetamiento molecular en estas películas [34-36], lo cual produce una disminución no-lineal del volumen de los espacios libres que existen a nivel intermolecular (Vh, Figura 5).

Este comportamiento permite sugerir que el mecanismo de relajación estructural se vería alterado de forma significativa debido a la reducción del volumen libre que ejerce el sorbitol sobre la matriz en estado vítreo y al aumento en el grado de frustración molecular de las cadenas de gelatina en presencia de sorbitol en sistemas de movilidad molecular reducida $[33,34,50]$.

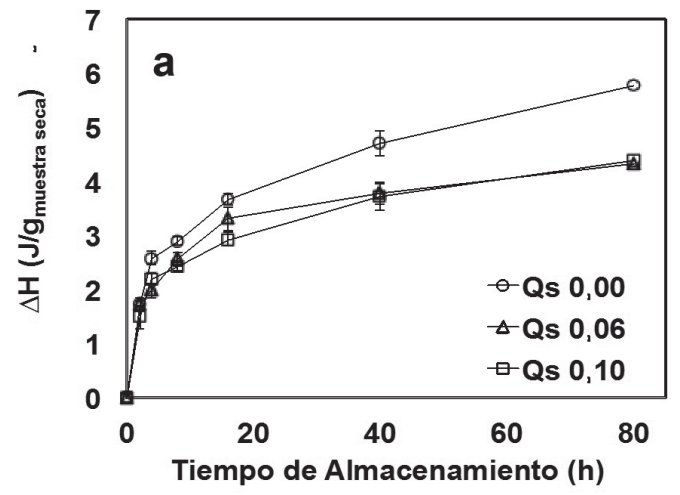

Como se ha señalado, durante el proceso de relajación, la matriz reduciría su densidad (disminución del volumen específico, Figura 1), pero de acuerdo al incremento en el empaquetamiento molecular asociado a la presencia de sorbitol, se estarían generando condiciones de impedimento físico a nivel molecular que alterarían de forma importante el mecanismo de relajación estructural (densificación) afectando directamente a la cinética de relajación la cual, bajo estas condiciones, se desarrolla a una cinética reducida (Figura 4). Sin embargo tal como ha sido señalado previamente, no se observaron diferencias significativas en la cinética de relajación a distintos niveles de sorbitol, lo cual indicaría que el fenómeno de relajación no sólo estaría siendo afectado por los cambios nano estructurales asociados con el aumento del empaquetamiento molecular por efecto de la presencia de sorbitol, sino que también por otros factores entre los cuales se podrían considerar el grado de interacción sorbitol-gelatina o la existencia de micro-zonas o clusters ricos en sorbitol dentro de la matriz.

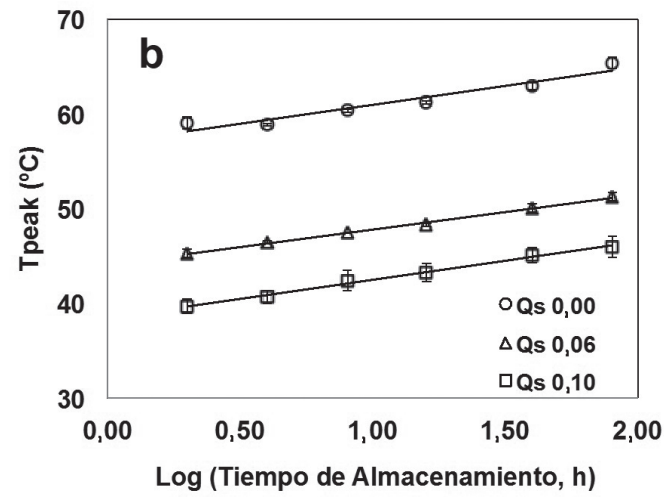

Figura 3. (a) Entalpía de relajación $\left(\Delta \mathrm{H}, \mathrm{J} / \mathrm{g}_{\text {muestra seca }}\right)$ en películas de gelatina-sorbitol en función del tiempo de almacenamiento, y (b) variación del valor de temperatura asociado al peak de la endoterma de relajación en películas de gelatina-sorbitol en función del logaritmo del tiempo de almacenamiento ( $R^{2}>0,95$ en todos los casos).

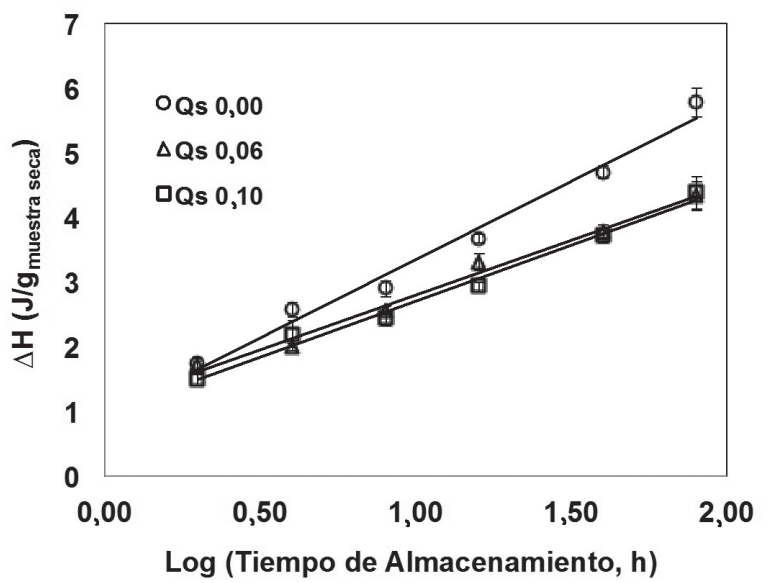

Figura 4. Entalpía de relajación $\left(\Delta \mathrm{H}, \mathrm{J} / \mathrm{g}_{\text {muestra seca }}\right)$ en películas de gelatina-sorbitol en función del logaritmo del tiempo de almacenamiento. 


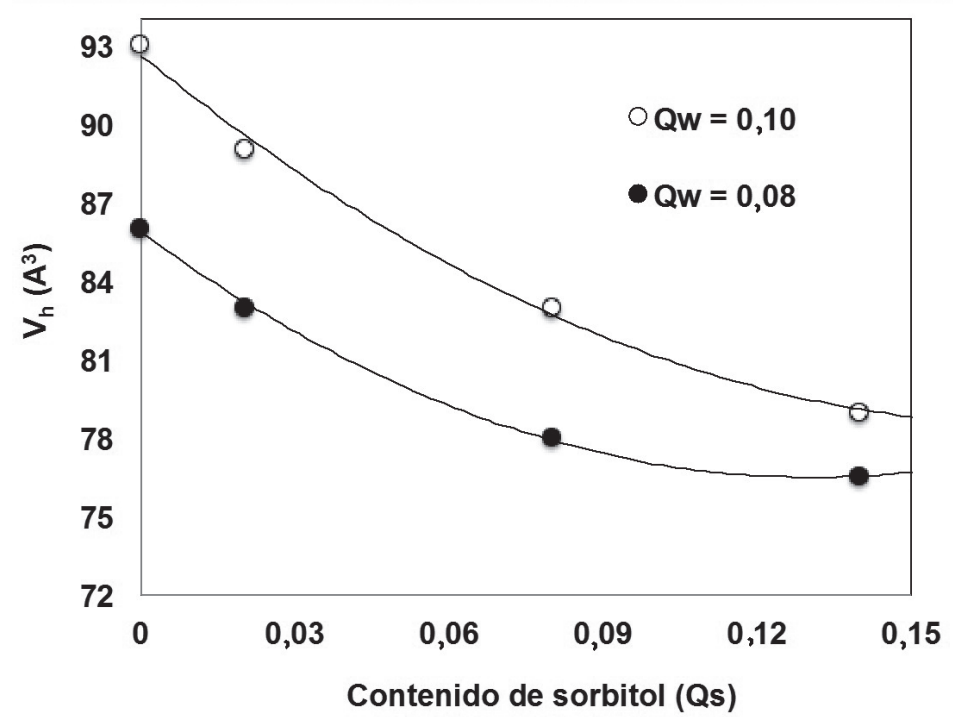

Figura 5. $V_{h}$ en función del contenido de sorbitol a dos contenidos de humedad bien definidos $\left(20^{\circ} \mathrm{C}\right)[50]$.

Esta modificación nano-estructural de la matriz polimérica en estado vítreo por efecto de plastificantes junto con modificar la cinética de relajación estructural tendría además un efecto importante sobre las propiedades de transporte, especialmente sobre la difusividad. En efecto, se ha establecido que la movilidad molecular y las propiedades de barrera en matrices poliméricas están directamente relacionadas con el volumen libre existente a nivel intermolecular $[51,52]$. El sorbitol debido a su bajo peso molecular y a la capacidad que tiene de incrementar el empaquetamiento molecular de la matriz, modificaría la difusividad del sistema gracias a la capacidad que posee de reducir el tamaño de los espacios libres intermoleculares (Vh, Figura 5), modificando de esta forma la transferencia de masa a través de la matriz. Esto ocurriría en paralelo a la capacidad del sorbitol de reducir la movilidad molecular del sistema debido a su reconocida actividad plastificante (Tabla 1). Este fenómeno reviste importancia tecnológica cuando por ejemplo la gelatina se utiliza como material encapsulante para proteger a ciertos compuestos funcionales del contacto con el oxígeno. Por lo tanto, estos resultados abren interesantes perspectivas de aplicación tecnológica al sugerir que la presencia de sorbitol modificaría la organización estructural del sistema a escala molecular, lo que permitiría modular la difusividad y la estabilidad estructural del material, lo cual es relevante en el diseño óptimo de matrices poliméricas vítreas utilizadas para la encapsulación de compuestos bioactivos, funcionales $y / 0$ nutracéuticos sensibles al oxígeno, como también en sistemas de liberación controlada.

\section{Conclusiones}

La presencia de sorbitol reduce significativamente el contenido de humedad y el valor de $\mathrm{Tg}$ de películas de gelatina de bovino en estado vítreo, y modifica significativamente la cinética de relajación estructural en películas de gelatina almacenadas $10^{\circ} \mathrm{C}$ bajo $\mathrm{Tg}$, reduciendo el valor de la entalpía de relajación $(\Delta \mathrm{H})$ en función del tiempo de almacenamiento. Sin embargo, no fue posible establecer diferencias significativas en el contenido de humedad y en la entalpía de relajación con los niveles de sorbitol estudiados. La reducción de la cinética de relajación por efecto del sorbitol se hace evidente al comparar el valor de la pendiente de la curva obtenida al representar los datos en escala semi-logarítmica. Este fenómeno podría estar relacionado directamente con los cambios nano-estructurales reportados recientemente mediante espectroscopía de positrones (PALS), los cuales se asocian con el rol del sorbitol que en el estado vítreo actúa aumentado el empaquetamiento molecular en matrices poliméricas. Este fenómeno tendría implicaciones tecnológicas relevantes en el área de la encapsulación de compuestos funcionales y en el diseño de sistemas de liberación controlada. 


\section{Agradecimientos}

Los autores agradecen el financiamiento otorgado por los proyectos FONDECYT N01110607 y PAI No79130039.

\section{Referencias bibliográficas}

[1] Slade L, Levine H. Glass transition and waterfood structure interactions. En: Advances in food and nutrition research. Kinsella JE, Editor. Estados Unidos: Academic Press. 1995;38:103-269.

[2] Roos YH. Glass transition temperature and its relevance in food processing. Ann. Rev. Food Sci. Technol. 2010;1:469-96.

[3] Roos YH, Karel J. Water and molecular weight effects on glass transitions in amorphous carbohydrates and carbohydrate solutions. J. Food Sci. 1991;56:1676-81.

[4] Roos YH. Phase Transitions in Foods. Estados Unidos: Academic Press Inc; 1995.

[5] Sperling LH. Introduction to Physical Polymer Science. 3 ed. Estados Unidos: Wiley; 1986.

[6] Roos YH, Karel M, Kokini JL. Glass transition in low moisture and frozen foods: effects on shelf life and quality. Food Technol-Chicago. 1996;50:95-108.

[7] Rahman MS. State diagram of foods: its potential use in food processing and product stability. Trends Food Sci. Tech. 2006;17:12941.

[8] Liu SB, Bhandari B, Zhou W. Glass transition and enthalpy relaxation of amorphous foods saccharides: A review. J. Agric. Food Chem. 2006;54:5701-17.

[9] Le Meste M, Champion D, Roudaut G, Blond G, Simatos D. Glass transition and food technology: A critical appraisal. J. Food Sci. 2002;67:2444-58.

[10] Andreozzi L, Faetti M, Giordano M, Palazzuoli D, Zulli F. Enthalpy Relaxation in Polymers: A Comparison among Different Multiparameter Approaches Extending the TNM/AGV Model. Macromolecules. 2003;36:7379-87.

[11] Andreozzi L, Faetti M, Giordano M, Zulli F. Molecular-Weight Dependence of Enthalpy Relaxation of PMMA. Macromolecules. 2005;38:6056-67.

[12] Ettiene S, Hazeg N, Duval E, Mermet A, Wypych A, David L. Physical aging and molecular mobility of amorphous polymers. J. Non-Cryst. Solids. 2007;353:3871-8.
[13] Berens AR, Hodge IM. Effects of Annealing and Prior History on Enthalpy Relaxation in Glassy Polymers. 1.Experimental Study on Poly(vinyl chloride). Macromolecules. 1982;15:756-61.

[14]Hutchinson J. Physical Aging of Polymers. Prog. Polym. Sci. 1995;20:703-60.

[15] Perera DY. Physical aging of organic coatings. Prog. Org. Coat. 2003;47:61-76.

[16] Sbovoda R, Pustková P, Málek J. Structural relaxation of polyvinyl acetate (PVAc). Polymer. 2008;49:3176-85.

[17] Chung HJ, Lim S-T. Physical aging of glassy normal and waxy rice starches: thermal and mechanical characterization. Carbohydr. Polym. 2004;57:15-21.

[18] Badii F, Martinet C, Mitchell J, Farhat IA. Enthalpy and mechanical relaxation of glassy gelatin films. Food Hydrocolloid. 2006;20:87984.

[19]Enrione J, Sáez C, López D, Skurtys O, Acevedo C, Osorio F, et al. Structural relaxation of salmon gelatin films in the glassy state. Food Bioprocess Tech. 2012;5:2446-53.

[20] Lourdin D, Colonna P, Brownse GJ, Noel TR, Ring SG. Structural relaxation and physical ageing of starchy materials. Carbohydr. Res. 2002;337:827-33.

[21] Struik LCE. Physical aging in amorphous polymers and other materials. Holanda: Elsevier; 1978.

[22] Pekarski P, Hampe J, Böhm I, Brion HG, Kirchheim R. Effect of aging and conditioning on diffusion and sorption of small molecules in polymer glasses. Macromolecules. 2000;33:2192-9.

[23] Jin Kim Y, Hagiwara T, Kawai K, Suzuki T, Takai R. Kinetic process of enthalpy relaxation of glassy starch and effect of physical aging upon its water vapor permeability property. Carbohydr. Polym. 2003;53:289-96.

[24] Hu CC, Fu YJ, Hsiao SW, Lee KR, Lai JY. Effect of physical aging on the gas transport properties of poly(methyl methacrylate) membranes. J. Membr. Sci. 2007;303:29-36.

[25] Kilburn D, Claude J, Schweizer T, Alam A, Ubbink J. Carbohydrate polymers in amorphous states: an integrated thermodynamic and nanostructural investigation. Biomacromolecules. 2005;6:864-79.

[26] Townrow S, Roussenova M, Giardello MI, Alam A, Ubbink J. Specific volume - Hole volume correlations in amorphous carbohydrates: effect of temperature, molecular weight 
and water content. J. Phys. Chem. B. 2010;114:1568-78.

[27] Roussenova M, Murith M, Alam A, Ubbink J. Plasticization, antiplasticization and molecular packing in amorphous carbohydrate-glycerol matrices. Biomacromolecules. 2010;11:323747.

[28] Díaz P, Arratia C, Vásquez C, Osorio F, Enrione J. Effect of glycerol on water sorption of bovine gelatin films in the glassy state. Procedia Food Sci. 2011;1:267-74.

[29] Ferry JD. Viscoelastic Properties of Polymers. 2 ed. Estados Unidos: Wiley; 1980.

[30] Lillie MA, Gosline JM. The effect of hydration on the dynamic mechanical properties of elastin. Biopolymers. 1990;29:1147-60.

[31] Harrison STL, Chase HA, Amor SR, Bhonthrone KM, Sanders JKM. Plasticization of poly(hydroxybutyrate) in vivo. Int. J. Biol. Macromol. 1992;14:50-6.

[32] Kilburn D, Claude J, Mezzenga R, Dlubek G, Alam A, Ubbink J. Water in glassy carbohydrates: opening it up at the nanolevel. J. Phys. Chem. B. 2004;108:12436-41.

[33] Townrow S, Kilburn D, Alam A, Ubbink J. Molecular packing in amorphous carbohydrate matrixes. J. Phys. Chem. B. 2007;111:1264348.

[34]Roussenova M, Enrione J, Díaz-Calderón P, Taylor AJ, Ubbink J, Alam MA. A nanostructural investigation of glassy gelatin oligomers: molecular organization and interactions with low molecular weight diluents. New J. Phys. 2012;14:35016-36.

[35] Roussenova M, Hughes DJ, Enrione J, DíazCalderón P, Sivaniah E, Song Q, et al. Free volume, molecular mobility and polymer structure: Towards the rational design of multi-functional materials. Acta Phys. Pol. A. 2014;125:801-5.

[36] Roussenova M, Enrione J, Díaz-Calderón P, Taylor AJ, Ubbink J, Alam MA. Effect of polyol on the molecular organization and thermodynamic properties of low water content gelatin oligomers. Polymer. 2014;55(26):682736.

[37] Enrione J, Hill S, Mitchell J. Sorption and diffusional studies of extruded waxy maize starch-glycerol systems. Starch/Stärke. 2007;59:1-9

[38] Enrione J, Hill S, Mitchell J. Sorption behaviour of mixtures of glycerol and starch. J. Agric. Food Chem. 2007;55:2956-63.
[39] Ubbink J, Giardello MI, Limbach HJ. Sorption of water by mixtures of carbohydrates in glassy and rubbery states. Biomacromolecules. 2007;8:2862-73.

[40] Talja RA, Helén H, Roos YH, Jouppila K. Effect of various polyols and polyol contents on physical and mechanical properties of potato starch-based films. Carbohydr. Polym. 2007;67:288-95.

[41] Surana R, Pyne A, Suryanarayanan R. Effect of aging on the physical properties of amorphous trehalose. Pharm. Res. 2004;21:867-74.

[42] Badii F, MacNaughtan W, Farhat IA. Enthalpy relaxation of gelatin in the glassy state. Int. J. Biol. Macromol. 2005;36:263-9.

[43] Tao SJ. Positron annihilation in molecular substances. J. Chem. Phys. 1972;56:5499510.

[44] Eldrup M, Lightbody D, Sherwood JN. The temperature dependence of positron lifetimes in solid pivalic acid. Chem. Phys. 1981;63:51-8.

[45] Lourdin D, Coignard H, Bizot H, Colonna P. Influence of equilibrium relative humidity and plasticizer concentration on the water content and glass transition of starch materials. Polymer. 1997;38:5401-6.

[46] Arvannitoyanis I, Psomiadou E, Nakayama A, Aiba S, Yamamoto N. Edible films made from gelatin, soluble starch and polyols, Part 3. Food Chem. 1997;60:593-604.

[47] Pouplin M, Redl A, Gontard N. Glass Transition of Wheat Gluten Plasticized with Water, Glycerol or Sorbitol. J. Agric. Food Chem. 1999;47:538-43.

[48] Cao N, Yang Y, Fu Y. Effects of various plasticizers on mechanical and water vapor barrier properties of gelatin films. Food Hydrocolloid. 2009;23:729-35.

[49] Badii F. Stability of proteins in the glassy state. Nottingham, Reino Unido: The University of Nottingham; 2005.

[50] Roussenova M. Molecular organisation and mobility in glass forming systems - A free volume perspective (tesis doctoral). Bristol, Reino Unido: University of Bristol; 2011.

[51] Vrentas JS, Duda JL. Diffusion in PolymerSolvent Systems. I. Re-examination of the Free-Volume Theory. J. Polym. Sci. 1977;15:403-16.

[52] Cohen MH, Grest GS. Liquid-glass transition, a free volume approach. Phys. Rev. B. 1979;20:1077-98. 